YAMAGATA-HEP-93-13

August 1993

\title{
Gaugeon Formalism with BRST Symmetry
}

\author{
Minoru KOSEKI, Masaaki SATO* and Ryusuke ENDO** \\ Graduate School of Science and Technology \\ Niigata University, Niigata 950-21, Japan \\ *Hitachi Tohoku Software Co., Sendai 980, Japan \\ ** Department of Physics, Yamagata University, Yamagata 990, Japan
}

\begin{abstract}
We provide a BRST symmetric version of Yokoyama's Type I gaugeon formalism for quantum electrodynamics; the similar theory by Izawa can be considered as a BRST symmetrized Type II theory. With the help of the BRST symmetry, Yokoyama's physical subsidiary conditions are replaced by the Kugo-Ojima type condition. As a result, the formalism becomes applicable even in the background gravitational field. We show how the Hilbert spaces of standard formalism in various gauges are embedded in the single Hilbert space of the present formalism. We also give a path integral derivation of the Lagrangian.
\end{abstract}

\footnotetext{
${ }^{\dagger}$ Preliminary result was reported at Niigata Autumn School on Particle Physics held on October 23-25, 1992, in Kusatsu.
} 


\section{Introduction}

In the standard formalism of canonically quantized gauge theories [1], 2] we cannot consider the gauge transformation freely. There exists no gauge freedom in the quantum theory, since the theory is defined only after the gauge fixing. Namely, a Hilbert space defined in a particular gauge is quite different from those in other gauges. Thus, if we want to realize the quantum gauge freedom, we need a wider Hilbert space.

Yokoyama's gaugeon formalism [3] - 9] provides a wider framework in which we can consider the quantum gauge transformation among a family of Lorentz covariant linear gauges. In this formalism a set of extra fields, so called gaugeon field, is introduced as the quantum gauge freedom. This theory was first proposed for the quantum electrodynamics [3] - 5] to resolve the problem of gauge parameter renormalization [10]. It was also applied later to the Yang-Mills theory [6, 9]. Thanks to the quantum gauge freedom of this formalism, the gauge parameter independence of the physical $S$-matrix becomes manifest [7]. It has also been shown, with the help of certain conjecture, that the wave-function renormalization constant is gauge independent in this formalism [8].

The extra gaugeon modes should be removed from the physical Hilbert space since the quantum gauge freedom is unphysical mode. In fact the gaugeon exhibits dipole character and yields negative normed states. To remove these modes Yokoyama imposed the Gupta-Bleuler type subsidiary condition [3]. However, this type of condition does not work well if interaction is present for the gaugeon field. Especially, we cannot use Yokoyama's subsidiary condition in the background gravitational field.

In the present paper we improve the subsidiary conditions of Yokoyama's formalism for the quantum electrodynamics. For this purpose we introduce extra Faddeev-Popov [FP] ghosts for the gaugeon field and provide a fully BRST symmetric Lagrangian. By the help of BRST charge the subsidiary condition is simply replaced by the Kugo-Ojima type condition [2], which is known to work well even in the interacting case.

In connection with this program, we should refer to the work by Izawa [11. After completed our work, we became aware of his paper in which he also provided a BRST symmetric gaugeon formalism and the Kugo-Ojima type subsidiary condition. The rela- 
tion of our theory to his is also discussed in this paper.

The paper is organized as the following. In $\S 2$ we briefly review Yokoyama's gaugeon formalism for quantum electrodynamics. In $\S 3$ a BRST symmetrized version of this formalism is proposed. And its relation to Izawa's theory is discussed. In $\S 4$ we see how the Hilbert space of the standard formalism is embedded in the wider space of the BRST symmetric gaugeon formalism. To confirm the necessity of FP ghosts for the gaugeon field, we re-derive our Lagrangian and Izawa's Lagrangian using path integral in $\S 5$. Section 6 is devoted to summary.

\section{Yokoyama's gaugeon formalism}

Yokoyama's Lagrangian for electromagnetic field $A_{\mu}$ interacting with charged matter field $\psi$ is given by

$$
\begin{aligned}
\mathcal{L}_{\mathrm{I}}= & -\frac{1}{4} F_{\mu \nu} F^{\mu \nu}+\partial_{\mu} B A^{\mu}+\partial_{\mu} Y_{*} \partial^{\mu} Y+\frac{\varepsilon}{2}\left(Y_{*}+\alpha B\right)^{2} \\
& -i \partial_{\mu} c_{*} \partial^{\mu} c+\mathcal{L}_{\text {matt }}\left(\psi, A_{\mu}\right)
\end{aligned}
$$

where $F_{\mu \nu}=\partial_{\mu} A_{\nu}-\partial_{\nu} A_{\mu}, B$ is the $B$-field of Nakanishi-Lautrup [1], $c$ and $c_{*}$ are usual FP ghosts, $Y$ and $Y_{*}$ are scalar fields called the gaugeon fields and its associated field respectively $\square$ and $\mathcal{L}_{\text {matt }}$ is the Lagrangian for the matter field $\psi$ minimally coupled to $A_{\mu}$. We have introduced here the FP ghosts $c$ and $c_{*}$ which did not appear in the original paper [3]. Yokoyama introduced them later in the application of his formalism to the Yang-Mills theory [6]. Namely, we start from the Abelian limit of Yokoyama's Lagrangian for the Yang-Mills field [6, 9] In (2.1), $\varepsilon$ denotes a sign factor $(\varepsilon= \pm 1)$ and $\alpha$ is a numerical gauge parameter. The gauge parameter of the standard formalism, which we denote by $a$ in the present paper, can be idetified with $a=\varepsilon \alpha^{2}$. For example, Yokoyama's Lagrangian gives the photon propagator as

$$
\frac{g_{\mu \nu}}{k^{2}}+(a-1) \frac{k_{\mu} k_{\nu}}{\left(k^{2}\right)^{2}} \quad \text { with } \quad a=\varepsilon \alpha^{2} .
$$

In particular, $\alpha=0$ corresponds to Landau gauge $(a=0)$ and $\alpha=1$ with $\varepsilon=+1$ gives Feynman gauge $(a=1)$. Note that the gaugeon sector in (2.1) decouples from the rest if

\footnotetext{
${ }^{1}$ We use field notation different from Yokoyama's; the fields $B, Y$ and $Y_{*}$ here are denoted by $B_{1}, B$ and $B_{2}$, respectively, in the original paper. [3]
} 
$\alpha=0$. Then the remaining sector has the same form with the Lagrangian of the standard formalism in Landau gauge.

The Lagrangian permits the q-number gauge transformation which enables us to vary gauge parameter. The transformation is defined by

$$
\begin{aligned}
& \hat{A}_{\mu}=A_{\mu}+\tau \partial_{\mu} Y, \\
& \hat{\psi}=e^{i \tau e Y} \psi, \\
& \hat{Y}_{*}=Y_{*}-\tau B, \\
& \hat{B}=B, \quad \hat{Y}=Y, \\
& \hat{c}=c, \quad \hat{c}_{*}=c_{*},
\end{aligned}
$$

with $\tau$ being a parameter of the transformation. Under this transformation the Lagrangian (2.1) is form-invariant, that is, it transforms as

$$
\mathcal{L}_{\mathrm{I}}\left(\phi^{A} ; \alpha\right)=\mathcal{L}_{\mathrm{I}}\left(\hat{\phi}^{A} ; \hat{\alpha}\right)
$$

where $\phi^{A}$ stands for any of the fields and $\hat{\alpha}$ is defined by

$$
\hat{\alpha}=\alpha+\tau \text {. }
$$

The form invariance (2.4) means that $\phi^{A}$ and $\hat{\phi}^{A}$ satisfy the same field equation except for the parameter $\alpha$ which should be replaced by $\hat{\alpha}$ for the $\hat{\phi}^{A}$ field equation. In this sense, we can shift the gauge parameter by this transformation. For example, starting from any value of $\alpha$, we can always take $\alpha=0$ gauge where the theory is equivalent to the Landau-gauge standard formalism (plus free $Y-Y_{*}$ system).

As subsidiary conditions to confine the unphysical modes, Yokoyama [6] adopted

$$
\begin{aligned}
& \left.Q_{\mathrm{B}(\mathrm{KO})} \mid \text { phys }\right\rangle=0, \\
& \left.Y_{*}^{(+)}(x) \mid \text { phys }\right\rangle=0,
\end{aligned}
$$

where $Q_{\mathrm{B}(\mathrm{KO})}$ is the usual BRST charge [2] and $Y_{*}^{(+)}(x)$ is the positive frequency part of $Y_{*}(x)$. The first condition is the usual one of the standard formalism, which confine the unphysical photons by the quartet mechanism [2]. The second removes the gaugeon 
modes. It is essential in the second condition that the $Y_{*}$ field satisfies the free field equation?

$$
\square Y_{*}=0 .
$$

If this does not hold, the positive frequency part $Y_{*}^{(+)}$becomes ambiguous and the condition (2.7) contradicts with time evolution in general. For example, we cannot use the condition (2.7) in the background curved space-time.

There is another type of Lagrangian for the gaugeon formalism. In Ref. [4] Yokoyama and Kubo discussed more general Lagrangians that include $B, Y$ and $Y_{*}$ fields. Their conclusion is that there are only two types of the theory which admits the q-number gauge transformation. The first type [Type I] is described by (2.1) and the the second [Type II] by

$$
\mathcal{L}_{\mathrm{II}}=-\frac{1}{4} F_{\mu \nu} F^{\mu \nu}+\partial_{\mu} B A^{\mu}+\frac{\alpha}{2} B^{2}+\partial_{\mu} Y_{*} \partial^{\mu} Y+\frac{1}{2} Y_{*} B+\cdots
$$

where the dots denote the matter sector and possible FP ghost term. The Lagrangian (2.9) is also form-invariant under the q-number gauge transformation (2.3). The $\alpha$ in $\mathcal{L}_{\text {II }}$ directly corresponds to the gauge parameter of the standard formalism: $a=\alpha$. We can shift this parameter into arbitrary value by the q-number gauge transformation. This should be compared with Type I theory in which we cannot change the sign of the gauge parameter $a=\varepsilon \alpha^{2}$. In the Type II case, however, the gaugeon sector would not decouple even if any value of the parameter $\alpha$ taken. Thus, the equivalence of the theory to the standard formalism is not so trivial as in the Type I theory.

\section{Gaugeon formalism with BRST symmetry}

We consider the Lagrangian

$$
\begin{aligned}
\mathcal{L}= & -\frac{1}{4} F_{\mu \nu} F^{\mu \nu}+\partial_{\mu} B A^{\mu}+\partial_{\mu} Y_{*} \partial^{\mu} Y+\frac{\varepsilon}{2}\left(Y_{*}+\alpha B\right)^{2} \\
& -i \partial_{\mu} c_{*} \partial^{\mu} c-i \partial_{\mu} K_{*} \partial^{\mu} K+\mathcal{L}_{\text {matt }}\left(\psi, A_{\mu}\right)
\end{aligned}
$$

\footnotetext{
${ }^{2}$ In the case of Yang-Mills field, $Y_{*}$ dose not satisfy the free field equation. Instead of it, however, the combination $Y_{*}+\alpha B$ satisfies the free field equation. And the condition (2.7) is replaced by

$$
\left(Y_{*}+\alpha B\right)^{(+)}|\mathrm{phys}\rangle=0
$$

in Yang-Mills case [6, 9]
} 
where we have introduced the FP ghosts $K$ and $K_{*}$ for the gaugeon fields. This Lagrangian is different from Yokoyama's (2.1) only in the term of $K$ and $K_{*}$ fields. Field equations which follow from (3.1) are

$$
\begin{aligned}
& \partial^{\mu} F_{\mu \nu}+\partial_{\nu} B+j_{\nu}=0, \\
& \partial^{\mu} A_{\mu}=\varepsilon \alpha\left(Y_{*}+\alpha B\right), \\
& \square Y=\varepsilon\left(Y_{*}+\alpha B\right), \\
& \square Y_{*}=0, \\
& \square c=\square c_{*}=0, \\
& \square K=\square K_{*}=0,
\end{aligned}
$$

with $j_{\mu}$ being the conserved current defined by $j_{\mu}=\partial \mathcal{L}_{\text {matt }} / \partial A^{\mu}$.

The Lagrangian (3.1) is invariant under the following BRST transformation,

$$
\begin{aligned}
& \delta_{\mathrm{B}} A_{\mu}=\partial_{\mu} c, \\
& \delta_{\mathrm{B}} \psi=i e c \psi, \\
& \delta_{\mathrm{B}} c_{*}=-i B, \\
& \delta_{\mathrm{B}} B=\delta_{\mathrm{B}} c=0, \\
& \delta_{\mathrm{B}} Y=K, \\
& \delta_{\mathrm{B}} K_{*}=-i Y_{*}, \\
& \delta_{\mathrm{B}} Y_{*}=\delta_{\mathrm{B}} K=0,
\end{aligned}
$$

which obviously satisfies the nilpotency, $\delta_{\mathrm{B}}{ }^{2}=0$. Because of the nilpotency, the BRST invariance is easily seen if we rewrite the Lagrangian as

$$
\begin{aligned}
\mathcal{L}= & -\frac{1}{4} F_{\mu \nu} F^{\mu \nu}+\mathcal{L}_{\text {matt }} \\
& +i \delta_{\mathrm{B}}\left[\partial_{\mu} c_{*} A^{\mu}+\partial_{\mu} K_{*} \partial^{\mu} Y+\frac{\varepsilon}{2}\left(K_{*}+\alpha c_{*}\right)\left(Y_{*}+\alpha B\right)\right] .
\end{aligned}
$$

BRST charge corresponding to this transformation is expressed by

$$
Q_{\mathrm{B}}=\int\left(c \overleftrightarrow{\partial_{0}} B+K \overleftrightarrow{\partial_{0}} Y_{*}\right) d^{3} x
$$


with $\overleftrightarrow{\partial_{0}}=\partial_{0}-\overleftarrow{\partial_{0}}$. By the help of this charge we can define the physical subspace $\mathcal{V}_{\text {phys }}$ as a space of states satisfying

$$
\left.Q_{\mathrm{B}} \mid \text { phys }\right\rangle=0 \text {. }
$$

This subsidiary condition removes the gaugeon modes as well as the unphysical photons from the physical subspace; $Y$ and $Y_{*}$ together with $K$ and $K_{*}$ constitute a BRST quartet.

We consider the following q-number gauge transformation:

$$
\begin{aligned}
& \hat{A}_{\mu}=A_{\mu}+\tau \partial_{\mu} Y, \\
& \hat{\psi}=e^{i \tau e Y} \psi, \\
& \hat{Y}_{*}=Y_{*}-\tau B, \\
& \hat{B}=B, \quad \hat{Y}=Y, \\
& \hat{c}=c+\tau K, \\
& \hat{K}_{*}=K_{*}-\tau c_{*}, \\
& \hat{c}_{*}=c_{*}, \quad \hat{K}=K,
\end{aligned}
$$

Under this field transformation, the Lagrangian is form-invariant:

$$
\mathcal{L}\left(\phi^{A}, \alpha\right)=\mathcal{L}\left(\hat{\phi}^{A}, \hat{\alpha}\right)
$$

with $\hat{\alpha}=\alpha+\tau$. Thus, $\hat{\phi}^{A}$ also satisfies the field equations (3.2)-(3.7) with $\alpha$ replaced by $\hat{\alpha}$.

It should be noted that the q-number gauge transformation ( 3.12$)$ commutes with the BRST transformation (3.8). As a result, our BRST charge (3.10) is invariant under the q-number transformation:

$$
\hat{Q}_{\mathrm{B}}=Q_{\mathrm{B}}
$$

The physical subspace $\mathcal{V}_{\text {phys }}$ is, therefore, invariant under the q-number gauge transformation:

$$
\hat{\mathcal{V}}_{\text {phys }}=\mathcal{V}_{\text {phys }}
$$

This situation does not occur in Yokoyama's partially BRST symmetric theory [6, 9]. Yokoyama's condition (2.7) is not invariant under the transformation (2.3). 
Before concluding this section, we refer to Izawa's theory [11]. He has given a BRST symmetric Lagrangian which admits the q-number gauge transformation. His Lagrangian is similar to ours, but slightly different. Actually, it can be expressed in our notation as

$$
\mathcal{L}_{\text {Izawa }}=\mathcal{L}_{\text {II }}-i \partial_{\mu} K_{*} \partial^{\mu} K
$$

Namely, Izawa's Lagrangian can be regarded as a BRST symmetric version of the Type II gaugeon theory, [䧃] while ours is of Type I. Especially, one cannot decouple the gaugeon sector from the Lagrangian by choosing any value for the gauge parameter. Thus, its equivalence to the standard formalism in the Landau gauge is not so manifest as that of the Type I theory.[

\section{Gauge structure of Hilbert space}

As well as the BRST symmetry (3.8), the Lagrangian (3.1) has several other symmetries. For example, we have the following BRST-like conserved charges:

$$
\begin{aligned}
& Q_{\mathrm{B}(\mathrm{KO})}=\int c \stackrel{\leftrightarrow}{\partial_{0}} B d^{3} x, \\
& Q_{\mathrm{B}(\mathrm{Y})}=\int K \overleftrightarrow{\partial_{0}} Y_{*} d^{3} x, \\
& Q_{\mathrm{B}(\mathrm{KO})}^{\prime}=\int K \overleftrightarrow{\partial_{0}} B d^{3} x, \\
& Q_{\mathrm{B}(\mathrm{Y})}^{\prime}=\int c \stackrel{\leftrightarrow}{\partial_{0}} Y_{*} d^{3} x .
\end{aligned}
$$

The charge $Q_{\mathrm{B}(\mathrm{KO})}$ generates the BRST transformation for $A_{\mu}, B, c, c_{*}$ and $\psi$ fields, that is, the same BRST transformation as in the standard formalism. The transformation generated by $Q_{\mathrm{B}(\mathrm{Y})}$ is the BRST transformation only for $Y, Y_{*}, K$ and $K_{*}$ fields. Thus, $Q_{\mathrm{B}}$ in the last section can be decomposed as

$$
Q_{\mathrm{B}}=Q_{\mathrm{B}(\mathrm{KO})}+Q_{\mathrm{B}(\mathrm{Y})}
$$

The charge $Q_{\mathrm{B}(\mathrm{KO})}^{\prime}$ generates the BRST transformation for $A_{\mu}, B$ and $\psi$ but with $K$ and $K_{*}$ treated as their FP ghosts. Similarly, $Q_{\mathrm{B}(\mathrm{Y})}^{\prime}$ generates the BRST transformation for $Y$ and $Y_{*}$ with $c$ and $c_{*}$ as their FP ghosts.

\footnotetext{
${ }^{3}$ The equivalence will be seen in the next section.
} 
In last section, we have taken (3.11) as a physical subsidiary condition. Instead of it, however, we may choose the condition as

$$
\begin{aligned}
& \left.Q_{\mathrm{B}(\mathrm{KO})} \mid \text { phys }\right\rangle=0, \\
& \left.Q_{\mathrm{B}(\mathrm{Y})} \mid \text { phys }\right\rangle=0 .
\end{aligned}
$$

The unphysical photons are removed by the first condition, while the gaugeon modes by the second. Thus, this pair of conditions is much similar to Yokoyama's pair (2.6) and (2.7) than the single condition (3.11). We denote the space of states satisfying (4.3) by $\mathcal{V}_{\text {phys }}^{(\alpha)}$. As easily seen, this space is a subspace of $\mathcal{V}_{\text {phys }}$ defined in last section,

$$
\mathcal{V}_{\text {phys }}^{(\alpha)} \subset \mathcal{V}_{\text {phys }}
$$

We have used the index $(\alpha)$ in $\mathcal{V}_{\text {phys }}^{(\alpha)}$ to emphasize that the definition of the subspace $\mathcal{V}_{\text {phys }}^{(\alpha)}$ depends on the gauge parameter $\alpha$. In fact, the BRST charges $Q_{\mathrm{B}(\mathrm{KO})}$ and $Q_{\mathrm{B}(\mathrm{Y})}$ are not invariant under the q-number gauge transformation (3.12). They transforms as

$$
\begin{aligned}
& \hat{Q}_{\mathrm{B}(\mathrm{KO})}=Q_{\mathrm{B}(\mathrm{KO})}+\tau Q_{\mathrm{B}(\mathrm{KO})}^{\prime}, \\
& \hat{Q}_{\mathrm{B}(\mathrm{Y})}=Q_{\mathrm{B}(\mathrm{Y})}-\tau Q_{\mathrm{B}(\mathrm{KO})}^{\prime},
\end{aligned}
$$

while their sum $Q_{\mathrm{B}}$ (and thus $\mathcal{V}_{\text {phys }}$ ) remains invariant. In the following we show that the space $\mathcal{V}_{\text {phys }}^{(\alpha)}$ has the same structure with those of the physical subspace of the standard formalism with the gauge parameter $a=\varepsilon \alpha^{2}$. In particular, all of the Green functions for physical operators agree.

First we define a subspace $\mathcal{V}^{(\alpha)}$ of the total space $\mathcal{V}$ by

$$
\mathcal{V}^{(\alpha)}=\left\{|\Phi\rangle \in \mathcal{V} ; Q_{\mathrm{B}(\mathrm{Y})}|\Phi\rangle=0\right\} \subset \mathcal{V},
$$

which includes $\mathcal{V}_{\text {phys }}^{(\alpha)}$ as a subspace since $\mathcal{V}_{\text {phys }}^{(\alpha)}$ can be expressed as

$$
\mathcal{V}_{\text {phsy }}^{(\alpha)}=\left\{|\Phi\rangle \in \mathcal{V}^{(\alpha)} ; Q_{\mathrm{B}(\mathrm{KO})}|\Phi\rangle=0\right\} \subset \mathcal{V}^{(\alpha)}
$$

by definition. The space $\mathcal{V}^{(\alpha)}$ corresponds to the total space of the standard formalism in the $a=\varepsilon \alpha^{2}$ gauge. And thus, as seen from (4.7), $\mathcal{V}_{\text {phys }}^{(\alpha)}$ corresponds to the physical subspace of the $a=\varepsilon \alpha^{2}$ standard formalism. To see this we notice the following three facts: 
1. The equal-time commutation relations for the fields $A_{\mu}, B, c, c_{*}$ and $\psi$ are exactly the same with those of the standard formalism.

2. If we take the matrix elements among the states of $\mathcal{V}^{(\alpha)}, A_{\mu}, B, c, c_{*}$ and $\psi$ satisfy the same field equations with those for the standard formalism in the $\varepsilon \alpha^{2}$-gauge. For example,

$$
\left\langle\Phi_{1}\left|\partial^{\mu} A_{\mu}-\varepsilon \alpha^{2} B\right| \Phi_{2}\right\rangle=0, \quad \text { for }\left|\Phi_{1}\right\rangle,\left|\Phi_{2}\right\rangle \in \mathcal{V}^{(\alpha)}
$$

which is easily seen if we rewrite the equation (3.3) as

$$
\partial^{\mu} A_{\mu}-\varepsilon \alpha^{2} B=i \varepsilon \alpha\left\{Q_{\mathrm{B}(\mathrm{Y})}, K_{*}\right\}
$$

3. Any state given by a product of the field operators $A_{\mu}, B, c, c_{*}$ and $\psi$ acting on the vacuum state is included in $\mathcal{V}^{(\alpha)}$, since these fields are $Q_{\mathrm{B}(\mathrm{Y}) \text {-singlets. }}$

The second fact can be easily understood if we express the Lagrangian (3.1) as

$$
\mathcal{L}=\mathcal{L}_{\mathrm{KO}}^{\left(a=\epsilon \alpha^{2}\right)}+\left\{i Q_{\mathrm{B}(\mathrm{Y})}, \partial_{\mu} K_{*} \partial^{\mu} Y+\frac{\varepsilon}{2} K_{*}\left(Y_{*}+\alpha B\right)\right\}
$$

where $\mathcal{L}_{\mathrm{KO}}^{\left(a=\epsilon \alpha^{2}\right)}$ denotes the Lagrangian of the standard formalism in $a=\varepsilon \alpha^{2}$ gauge. The facts 1 and 2 mean that the field equations and the (four-dimensional) commutation relations are the same with those of the standard formalism in $\varepsilon \alpha^{2}$ gauge if their matrix elements are assumed to be taken in $\mathcal{V}^{(\alpha)}$. Combining this with 3 , we can conclude that any vacuum expectation value of the products of $A_{\mu}, B, c, c_{*}$ and $\psi$ fields coincides with that evaluated in the standard formalism.

It should be noted that the discussion in this section also applies to Izawa's theory. Especially, his Lagrangian can be written as

$$
\mathcal{L}_{\text {Izawa }}=\mathcal{L}_{\mathrm{KO}}^{(a=\alpha)}+\left\{i Q_{\mathrm{B}(\mathrm{Y})}, \partial_{\mu} K_{*} \partial^{\mu} Y+\frac{1}{2} K_{*} B\right\}
$$

which leads to, for example, the equation similar to (4.8). 


\section{Path integral}

We start from the gauge invariant path integral

$$
\begin{aligned}
& Z=\int \mathcal{D} A_{\mu} \exp \left[i \int \mathcal{L}_{0} d^{4} x\right] \\
& \mathcal{L}_{0}=-\frac{1}{4} F^{\mu \nu} F_{\mu \nu}
\end{aligned}
$$

where we have omitted the matter field $\psi$ for simplicity since its presence is not essential in the following discussion. Before factoring out the group volume by Faddev-Popov's trick [12], we multiply $Z$ by unit

$$
1=\operatorname{det} \square^{-1} \cdot \operatorname{det} \square
$$

and express the functional determinants as

$$
\begin{aligned}
\operatorname{det} \square^{-1} & =\int \mathcal{D} Y_{*} \mathcal{D} Y \exp \left[i \int \mathcal{L}_{\mathrm{Y}} d^{4} x\right], \\
\operatorname{det} \square & =\int \mathcal{D} K_{*} \mathcal{D} K \exp \left[i \int \mathcal{L}_{\mathrm{K}} d^{4} x\right],
\end{aligned}
$$

with

$$
\begin{aligned}
& \mathcal{L}_{\mathrm{Y}}=\partial_{\mu} Y_{*} \partial^{\mu} Y+\frac{\alpha_{1}}{2} Y_{*}^{2}, \\
& \mathcal{L}_{\mathrm{K}}=\partial_{\mu} K_{*} \partial^{\mu} K,
\end{aligned}
$$

where $Y$ and $Y_{*}$ are bosonic scalar variables and $K$ and $K_{*}$ fermionic scalar variables. In (5.4), $\alpha_{1}$ is a numerical parameter; f a similar $K_{*}^{2}$-term cannot be introduced in $\mathcal{L}_{\mathrm{K}}$ because of the anticommuting character of $K$ and $K_{*}$. The gauge invariant path integral (5.1) is now

$$
Z=\int \mathcal{D} A_{\mu} \mathcal{D} Y_{*} \mathcal{D} Y \mathcal{D} K_{*} \mathcal{D} K \exp \left[i \int\left(\mathcal{L}_{0}+\mathcal{L}_{\mathrm{Y}}+\mathcal{L}_{\mathrm{K}}\right) d^{4} x\right]
$$

We consider here the following gauge condition

$$
\mathcal{F} \equiv \partial^{\mu} A_{\mu}-\alpha_{2} Y_{*}=\mathcal{C}(x)
$$

\footnotetext{
${ }^{4}$ Although $\alpha_{1}$ is an arbitrary parameter, we can assume $\alpha_{1}=\varepsilon= \pm 1$ or 0 without any loss of generality, since we may always use the field redefinitions $Y_{*} \rightarrow \pm e^{\lambda} Y_{*}$ and $Y \rightarrow \pm e^{-\lambda} Y$ which do not change the kinetic term in $\mathcal{L}_{\mathrm{Y}}$.
} 
where $\alpha_{2}$ is a numerical parameter and $\mathcal{C}(x)$ is an arbitrary given function. Using FaddeevPopov's trick for this gauge condition, we get

$$
\begin{aligned}
Z= & \int \mathcal{D} A_{\mu} \mathcal{D} B \mathcal{D} Y_{*} \mathcal{D} Y \mathcal{D} K_{*} \mathcal{D} K \Delta_{\mathrm{FP}} \\
& \times \exp \left[i \int\left\{\mathcal{L}_{0}+\mathcal{L}_{\mathrm{Y}}+\mathcal{L}_{\mathrm{K}}-B(\mathcal{F}-\mathcal{C})\right\} d^{4} x\right],
\end{aligned}
$$

where we have expressed the $\delta$-functional $\delta[\mathcal{F}-\mathcal{C}]$ in the Fourier transformation form (integrated over $B$ ) and $\Delta_{\mathrm{FP}}$ is the usual Faddeev-Popov determinant. Since the left hand side is independent of the choice of the function $\mathcal{C}$, we can take the 't Hooft averaging [13] over $\mathcal{C}$. With the weight functional

$$
\exp \left[i \int \frac{1}{2 \alpha_{3}} \mathcal{C}(x)^{2} d^{4} x\right],
$$

we finally get

$$
Z=\int \mathcal{D} A_{\mu} \mathcal{D} B \mathcal{D} c_{*} \mathcal{D} c \mathcal{D} Y_{*} \mathcal{D} Y \mathcal{D} K_{*} \mathcal{D} K \exp \left[i \int \mathcal{L}_{\mathrm{YK}} d^{4} x\right]
$$

where

$$
\begin{aligned}
\mathcal{L}_{\mathrm{YK}}= & -\frac{1}{4} F_{\mu \nu} F^{\mu \nu}+\partial_{\mu} B A^{\mu}+\frac{\alpha_{3}}{2} B^{2}+\alpha_{2} B Y_{*}+\partial_{\mu} Y_{*} \partial^{\mu} Y \\
& +\frac{\alpha_{1}}{2} Y_{*}^{2}-i \partial_{\mu} c_{*} \partial^{\mu} c-i \partial_{\mu} K_{*} \partial^{\mu} K .
\end{aligned}
$$

Thus we get the Lagrangian with three parameters. Up to the FP ghost terms it coincides with the three-parameter Lagrangian discussed by Yokoyama and Kubo [4], the most general Lagrangian that includes the gaugeon fields. Note that the Lagrangian (5.13) is invariant under the BRST transformation (3.8). By choosing special values for these parameters, we get the Lagrangian of Type I if $\alpha_{1}= \pm 1=\varepsilon, \alpha_{2}=\varepsilon \alpha, \alpha_{3}=\varepsilon \alpha^{2}$, and of Type II if $\alpha_{1}=0, \alpha_{2}=1 / 2, \alpha_{3}=\alpha$. (For other choice of the parameters, see Ref. 四. )

We have thus obtained the BRST symmetric Lagrangians of Type I and of Type II (Izawa's Lagrangian) by path integral. The application to the curved space-time case is straightforward. From this derivation we easily understand the necessity of the gaugeon FP ghosts $K$ and $K_{*}$. Without these FP ghosts the path integral differs by det $\square$, which cannot be ignored in the background gravitational field. 


\section{Summary}

By introducing gaugeon FP ghosts $K$ and $K_{*}$, we have given a BRST symmetric version of Type I gaugeon formalism for quantum electrodynamics. We also pointed out that the similar theory by Izawa can be regarded as a BRST symmetrized Type II theory. The BRST symmetry enables us to improve Yokoyama's subsidiary conditions (2.6) and (2.7). We have replaced them by the single Kugo-Ojima type condition (3.11), so that the formalism becomes applicable to the case of background gravitational field.

Unlike Yokoyama's partially BRST symmetric theory, our fully symmetric theory has the physical subspace $\mathcal{V}_{\text {phys }}$ invariant under the q-number gauge transformation. We have seen in $\S 4$ that this gauge invariant space $\mathcal{V}_{\text {phys }}$ includes various gauge variant subspaces

$\mathcal{V}_{\text {phys }}^{(\alpha)}$, which can be identified with the physical subspaces of the standard formalism in $a=\varepsilon \alpha^{2}$ (or $\left.a=\alpha\right)$ gauge.

A path integral derivation of our Lagrangian and Izawa's one is presented in $§ 5$. The derivation shows that the gaugeon FP ghosts $K$ and $K_{*}$ are certainly necessary for the gaugeon formalism, especially in the background gravitational field.

In this paper we restrict ourselves to the Abelian gauge theory. We can also present BRST symmetric gaugeon formalism for the Yang-Mills theory, which will be reported in the forthcoming paper.

\section{Acknowledgements}

We would like to thank Professor K. Ishida for helpful comments and encouragement.

\section{References}

[1] N. Nakanishi, Prog. Theor. Phys. Suppl. No.51 (1972).

[2] T. Kugo and I. Ojima, Phys. Lett. 73B (1978), 459; Prog. Theor. Phys. 60 (1978), 1896; Prog. Theor. Phys. Suppl. No.66 (1979), 1.

[3] K. Yokoyama, Prog. Theor. Phys. 51 (1974), 1956.

[4] K. Yokoyama and R. Kubo, Prog. Theor. Phys. 52 (1974), 290. 
[5] K. Yokoyama, Quantum Electrodynamics (in Japanese) (Iwanami Shoten, Tokyo, 1978)

[6] K. Yokoyama, Prog. Theor. Phys. 59 (1978), 1699.

K. Yokoyama, M. Takeda and M. Monda, Prog. Theor. Phys. 60 (1978), 927.

K. Yokoyama, Prog. Theor. Phys. 60 (1978), 1167.

[7] K. Yokoyama, Phys. Lett. 79B (1978), 79.

[8] T. Fukuda, R. Kubo and K. Yokoyama, Prog. Theor. Phys. 63 (1980), 1384.

[9] K. Yokoyama, M. Takeda and M. Monda, Prog. Theor. Phys. 64 (1980), 1412.

[10] M. Hayakawa and K. Yokoyama, Prog. Theor. Phys. 44 (1970), 533.

[11] K. Izawa, Prog. Theor. Phys. 88 (1992), 759.

[12] L. D. Faddeev and V. N. Popov, Phys. Lett. 25B (1967), 29.

[13] G. 't Hooft, Nucl. Phys. B33 (1971), 173. 